Methods A staff survey was conducted to gain views and user experiences of comfort observations. This paper survey was disseminated to different wards by SPCT members or ward 'end of life care' champions.

Results There were 42 respondents from different wards representing a range of healthcare professionals. Qualitatively the survey showed that staff felt empowered by the comfort observations to proactively monitor dying patients; it enabled me to continue to monitor the patient without causing distress or discomfort'. They facilitated a structured approach and appropriate escalation. Staff felt patient care, and care of loved ones improved as a result; 'Early recognition of pain. Reason for agitation and can be treated without delay. Although EWS is stopped, the continuous monitoring of patient is possible via comfort obs and alert system. Better end of life care experience for patient and relatives'. 100\% of respondents felt the comfort obs were user friendly and would recommend them to other wards.

Conclusions Acute trusts are an important provider of end of life care. Comfort observations support staff to deliver proactive excellent end of life care through routine monitoring of domains of comfort and escalation of care when indicated. A robust training programme to support their use is being planned in addition to conducting a retrospective audit to assess if their use leads to improved frequency of assessing patient and carer needs.

\section{A UK-WIDE SERVICE EVALUATION OF DOCUMENTS RELATING TO ANTICIPATORY PRESCRIBING AT THE END OF LIFE}

Richella Ryan, Anna Spathis, Ben Bowers, Stephen Barclay. University of Cambridge

\subsection{6/bmjspcare-2019-ASP.72}

Background Anticipatory Prescribing (AP), or 'Just in Case' prescribing ahead of need, is a well-established component of end of life care in the UK. It aims to facilitate effective symptom control in the home and care home setting and reduce crisis hospital admissions near the end of life. However, it is a complex multi-system process with great potential for variation and error. The aim of this study is to investigate the role of local policies and procedures in guiding AP practice in community end of life care across the UK.

Methods The first (England) phase of a UK-wide service evaluation has sampled 55 CCGs in England, stratified by NHS local office region, size and level of urbanisation. A range of stakeholders (senior doctors, nurses, pharmacists or CCG managers) were contacted by email and asked to identify and supply the documents used in their areas to regulate and facilitate AP.

Results Responses obtained to date are from 29 of 55 CCG areas in England (53\%). Whilst all areas have some form of generic prescribing guidance, only $11 / 29$ (38\%) have specific AP policies, 10/27 (37\%) have AP-related patient information leaflets and 4/27 (15\%) have AP standard operating procedures.

Conclusion Despite the complexity of the AP process, there is limited formal documentation of standard procedures and professional responsibilities in many areas, with considerable variation in type and level of regulation. Data collection is continuing and will be extended to all four UK countires, with qualitative analysis of responses and documents supplied planned.

\section{0 \\ VOLATILE ORGANIC COMPOUNDS PREDICT THE LAST WEEK OF LIFE IN LUNG CANCER PATIENTS}

S Coyle, E Chapman, S Mason, D Hughes, M Boyd, C Probert, J Ellershaw. St Helen's and Knowsley Hospitals Trust, University of Liverpool, Royal Liverpool and Broadgreen University Hospitals NHS Trust

\subsection{6/bmjspcare-2019-ASP.73}

Background Recognising dying is difficult and is an ongoing difficulty for doctors. We believe there is a process to dying and animal studies support this theory. We previously demonstrated that a number of volatile organic compounds in urine, change in the last weeks and days of life of patients in a small mixed cancer group. We needed to verify this finding in a suitably powered follow-up study.

Method We prospectively collected urine samples from people with lung cancer. We aimed to compare samples from 25 people in each of the last 3 weeks of life to a control group, 50 people with lung cancer who lived 3 or more months from the time of sampling. The urine samples were analysed for volatile organic compounds by gas chromatography mass spectrometry (GC-MS).

Least Absolute Shrinkage and Selection Operator (LASSO) logistic regression was used to analyse the GC-MS data and create a statistical model.

Results We recruited 162 people in total; 29 in the last week; 28 in the second last week; 30 in the third last week of life; 74 controls i.e. samples taken $>3$ months from death; 424 urine samples.

A model was created to predict whether a patient would die within 1 week. It has an optimism corrected AUC of 0.851 (95\% CI: $0.767,0.911$ ); sensitivity 78.6\% (95\% CI: $(64.3 \%, \quad 89.3 \%))$; specificity $83.1 \% \quad(95 \%$ CI: $\quad(69.9 \%$, $92.3 \%))$. The model identified a selection of compounds that contributed to the identification of patients who were close to death.

Discussion

- The results confirm that volatile organic compounds can predict when people with lung cancer are in the last week of life.

- Our model to predict when a person with lung cancer is in the last week of life is approximately $80 \%$ accurate.

\section{HARD RESET INCREASES IDENTIFICATION OF PEOPLE AT THE END OF LIFE}

Carol Scholes, Jane Lawson, Marian Townsend. Herts Community NHS Trust

\subsection{6/bmispcare-2019-ASP.74}

Introduction Recognition that someone is entering the last year of life enables access to Palliative and End of Life Care (PEoLC) including advance care planning. There is currently under-identification of people in the last year of life. The NHS Community Trust covers a population of 1.1 million. Numbers of people identified as being in the last year of life have increased significantly since 2014, with strategies including staff education, use of the 'surprise question' and development and support of a PEoLC Champion in each service. However under-identification continued.

Aim To enable access to PEoLC through improved identification of the patients known to the Trust who are likely to be in the last year of life. 
Method In May 2018 there was a 'Hard Reset' week: senior trust management required the PEoLC Champion in each service to lead a review of the Systm1 clinical record of every adult on the community caseload and inpatients, using the surprise question and document the outcome on Systm1. They were supported by locality managers, clinical leads and specialist palliative care nurses. Data was captured from Systm1.

Results In May there was a considerable increase in number of people newly identified as being in the last year of life (857) which was most noticeable in non-cancer services, such as heart failure service (171) and speech and language therapy service (55). Overall there was a $43 \%$ increase in people newly identified in the three months following the hard restart (1065) compared with the three months before (745). Conclusion The Hard Reset method increased numbers of people identified as being in the last year of life which has been sustained several months later.

Next steps To sustain this improvement and ensure that those identified are able to access PEoLC.

\section{HOSPITAL ADMISSION, TREATMENT ESCALATION AND PREFERRED PLACE OF CARE FOR PATIENTS WITH HAEMATOLOGICAL MALIGNANCIES IN THEIR LAST YEAR OF LIFE}

Anna Grundy, Josh Wright, James Davies. Sheffield Teaching Hospitals

\subsection{6/bmjspcare-2019-ASP.75}

Background Haematological malignancies encompass a range of heterogeneous conditions. Despite treatment advances the prognosis of several patient cohorts remains poor. Such individuals more commonly receive active treatment in the weeks prior to death, require inpatient admission and die in hospital compared to those with non-haematological cancers. However, this has been shown to contradict patient expectations of end of life care.

Methods A retrospective audit of 30 deceased patients with haematological malignancies was conducted in a tertiary department. Patient notes informed the number of hospital admissions, clinician exploration of preferred place of care, treatment escalation decisions and involvement of palliative care services within the 12 months prior to death.

Results Patients died from their underlying malignancy in $83 \%$ of cases. At the time of death $97 \%$ had a DNACPR in situ; $55 \%$ of forms were signed in the 2 days prior.

Patients required a mean of 4 admissions. Dependence on supportive blood products, a higher incidence of neutropenic sepsis and aggressive treatments were several reasons thought to be accountable. Achievement of preferred place of care was associated with initiating these discussions earlier. Of the $26 \%$ of patients asked, none specified hospital. Involvement of palliative care services was highest in the 5 days before death; $40 \%$ of referrals were for end of life support.

Conclusions Clinicians recognise when patients with haematological malignancies are imminently approaching their last days of life. However, the repeated pattern of admission in those with a poor prognosis may represent missed opportunities to initiate earlier advance care planning which has been linked to an increase in achieving preferred place of care. Focus groups exploring the barriers to initiate such conversations and empower earlier communication was felt to be a more effective intervention than the introduction of a paper proforma. A subsequent re-audit to assess their value is recommended.

\section{INCREASING STAFF CONFIDENCE AND COMPETENCE TO DELIVER INDIVIDUALISED END OF LIFE CARE ON ELDERLY CARE WARDS: IMPLEMENTATION OF A SYMPTOMS OBSERVATION CHART AND CARE PLAN GUIDE}

Daisy Henderson, Irene Carey, Maggie Kennedy, Gemma O'Driscoll, Jasmine Quraishi, Roxanna Colebatch, Annabel Jones, Radhika Patel, Emily Thomas. Guy's and St Thomas' NHS Foundation Trust

\subsection{6/bmjspcare-2019-ASP.76}

Background Following the withdrawal of the Liverpool Care Pathway concerns were expressed about staff confidence and competence to deliver end of life care. In response to locally identified needs and the recommendations for individualised care plans underpinned by the Five Priorities for Care of the Dying Person we have designed and implemented a Quality Improvement Project(QIP) within our NHS Foundation Trust.

Method We have introduced two tools, a symptom observation chart and care plan guide modelled on the Five Priorities. The tools were piloted initially on an acute medical admissions ward and have now been cascaded across four elderly care wards. Implementation was tracked and supported using QI methodology to include Run charts and PDSA cycles. Our aim is that all patients on elderly care wards who were recognised as being in the last days of life have $60 \%$ of their nursing care plans completed in line with the Five Priorities and $5 / 7$ of symptoms listed on the symptom observation chart recorded and managed appropriately every 4 hours.

Results Over 22 weeks we identified 57 patients with End of Life Notifications. During this period $0 \%-70 \%$ of patients had $60 \%$ of their nursing care plans in line with the Five Priorities and $0 \%-100 \%$ of patients had 5/7 symptoms listed on the symptom observation chart recorded and managed appropriately every 4 hours. The variation in the use and completion of both tools may be explained to some extent by the small sample size and missing data $(59 \%$ of charts in PDSA1 and 50\% in PDSA2 were missing).

Conclusion Staff on these wards require ongoing education and efforts to promote engagement and investment in the tools. Data collection to assess the adherence to standards with regards to the timing of bedside assessments and escalation of concerns to senior staff where applicable is currently ongoing.

\section{THE VIEWS OF A DISTRICT GENERAL HOSPITAL COMMUNITY ON TALKING ABOUT END OF LIFE WISHES}

Natalie Wright, Fiona Power, Cheryl Morgan, Jane Samarsinghe, Sue Dargan, Clare Smith. Ashford and St Peter's NHS Foundation Trust

\subsection{6/bmispcare-2019-ASP.77}

Dying matters is a coalition which aims to help people talk more openly about death and dying. This includes raising awareness with the hope that more people will make plans for the end of life (EoL). As part of awareness week 2018 